\title{
Length-to-Depth Ratio Effects on Aerodynamic Surface Quantities of a Rarefied Hypersonic Gap Flow
}

\author{
Luis T. L. C. Paolicchi* and Wilson F. N. Santos ${ }^{\dagger}$ \\ National Institute for Space Research, Cachoeira Paulista, SP, 12630-000, BRAZIL
}

\begin{abstract}
Numerical simulations of two-dimensional steady-state hypersonic flow in a gap at different length-to-depth $(L / H)$ ratio are performed by using a Direct Simulation Monte Carlo (DSMC) method. The work focuses on the effects in the aerodynamic surface quantities, such as heat transfer, pressure and skin friction coefficients due to variations in the gap $L / H$ ratio. The analysis showed that aerodynamic surface quantities presented a large dependence on the $L / H$ ratio for the range investigated. It was found that heat transfer, pressure, and skin friction coefficients presented the maximum values along the gap downstream face, more precisely, at the vicinity of the gap shoulder. Furthermore, simulations showed that pressure and heating loads are several times larger than those for a smooth surface.
\end{abstract}

\section{Nomenclature}

a Speed of sound, $\mathrm{m} / \mathrm{s}$

A Area, $\mathrm{m}^{2}$

$C_{f} \quad$ Skin friction coefficient, Eq. (5)

$C_{h} \quad$ Heat transfer coefficient, Eq.( 1)

$C_{p} \quad$ Pressure coefficient, Eq. ( 3)

$c \quad$ Molecular velocity, $\mathrm{m} / \mathrm{s}$

d Molecular diameter, $\mathrm{m}$

$F_{N} \quad$ Ratio of real molecules to simulated molecules, dimensionless

$H \quad$ Gap depth, m

$K n$ Knudsen number, $\lambda / l$

$l \quad$ Characteristic length, $\mathrm{m}$

$L \quad$ Gap length, $\mathrm{m}$

$M \quad$ Mach number, $U / a$

$m \quad$ Molecular mass, $\mathrm{kg}$

$n \quad$ Number density, $\mathrm{m}^{-3}$

$N \quad$ Number of molecules

$N_{f} \quad$ Dimensionless number flux, $N / n_{\infty} U_{\infty}$

$p \quad$ Pressure, $\mathrm{N} / \mathrm{m}^{2}$

$q \quad$ Heat flux, $\mathrm{W} / \mathrm{m}^{2}$

Re Reynolds number, $U l / \mu$

$R \quad$ Reentry capsule nose radius, $\mathrm{m}$

$T$ Temperature, $\mathrm{K}$

$u, v \quad$ Normal and tangential velocity components, $\mathrm{m} / \mathrm{s}$

$U \quad$ Freestream velocity, $\mathrm{m} / \mathrm{s}$

$x, y \quad$ Cartesian axes in physical space, $\mathrm{m}$

$X \quad$ Dimensionless length, $x / \lambda_{\infty}$

$Y \quad$ Dimensionless height, $y / \lambda_{\infty}$

* Graduate Student, Combustion and Propulsion Laboratory.

${ }^{\dagger}$ Researcher, Combustion and Propulsion Laboratory, AIAA Member. 


$\begin{array}{ll}\chi & \text { Mass fraction, dimensionless } \\ \Delta t & \text { Time step, } \mathrm{s} \\ \lambda & \text { Molecular mean free path, } \mathrm{m} \\ \mu & \text { Viscosity, } \mathrm{Ns} / \mathrm{m}^{2} \\ \rho & \text { Density, } \mathrm{kg} / \mathrm{m}^{3} \\ \tau & \text { Shear stress, } \mathrm{N} / \mathrm{m}^{2} \\ \omega & \text { Viscosity index, dimensionless } \\ \text { Subscript } \\ d & \text { Refers to downstream surface } \\ H & \text { Refers to gap depth } H \\ L & \text { Refers to gap length } L \\ R & \text { Refers to rotational energy } \\ u & \text { Refers to upstream surface } \\ V & \text { Refers to vibrational energy } \\ w & \text { Refers to wall conditions } \\ \infty & \text { Refers to freestream conditions }\end{array}$

\section{Introduction}

SEPARATED and reattached supersonic flows have been under extensive interest in the last decades. These Stype of flows are frequent on surfaces of high-speed aerodynamic configurations, in which the performance is degraded by separated flows, and various components damaged due to intense heating loads at reattachment. Included in these flows are shock-boundary layer interactions, wakes, steps, cavity and gap flows. The presence of gaps in modern aerodynamics configurations occurs as a desired or undesired design feature. For instance, the thermal protection systems of the space shuttle orbiter require gaps between the used protection elements to account for thermal expansion. ${ }^{1,2}$ For the optimized design of gaps and protection elements, it is necessary to predict the flow conditions and thermal loads as accurate as possible. In the case of reentry vehicles, the boundary layer transition prediction is a requirement to define the thermal protection system. This protection is usually designed as an assembly of tiles. Gaps between the tiles may modify the boundary-layer state and eventually promote transition, inducing higher temperature levels than expected. ${ }^{1}$ Moreover, the gap flow structure can become even more complex if the gap dimensions are deformed by thermal or mechanical loads. ${ }^{3,4}$

A lot of experiments and numerical simulations ${ }^{1,2,3,4,5,6,7,8,9,10,11}$ have already been conducted to investigate the flowfield structure on gaps. The majority of these research studies on gaps has gone into considering laminar or turbulent flow over a wide range of Mach numbers in the continuum flow regime. For the purpose of this introduction, it will be sufficient to describe only a few of these studies.

Scott and Maraia ${ }^{2}$ have investigated the heating rate distribution and the temperature response on the gap walls of protection tiles. The analysis showed that the hottest location measured in the gap was at 0.75 inch from the upstream transverse gap, and the heat flux distribution in the gap was not constant in time, since the convective heating rate depended on the wall temperature of the gap. In addition, they have demonstrated that the heating rate depended on the gap width.

Hinderks et $\mathrm{al}^{3}$ have investigated the gap flow structure. They showed that exist a complex flow within the gap, consisting of a vortex superposed by an axial flow. The analysis showed that the heat flux transferred to the structure depends on the thermal state of the structure. Also, effects due to changes in the gap geometry caused by deformations in the gap structure demonstrated that deformations should be considered in the design analysis.

Traineau et al. ${ }^{11}$ found that an increase in the angle of attack from 10 to 15 degrees for the same case studied, generated a $48 \%$ increase in pressure at the surface and $22 \%$ increase in heat transfer. They also showed that increasing the depth and length of the gaps caused a significant increase in temperature. Furthermore, studies have found that three-dimensional effects are important to the size of the vertical flow zone at the top of the gap, where the 3-D size is much smaller than that in 2-D.

The extensive literature in this area is devoted primarily to research studies in the continuum flow regime. Nevertheless, there is little understanding of the physical aspects of hypersonic flow past to gaps related to the severe aerothermodynamic environment associated to a reentry vehicle. In this context, Paolicchi and Santos $^{12}$ have studied gaps situated in a rarefied hypersonic flow by employing the DSMC method. The 
work was motivated by the interest in investigating the length-to-depth $(L / H)$ ratio effects on the flowfield structure. The primary emphasis was to examine the behavior of the primary properties, such as velocity, density, pressure and temperature, due to changes on the gap $L / H$ ratio. It was observed the formation of only one vortex for $L / H$ ratio of $1,1 / 2,1 / 3$ and $1 / 4$. Results showed that gap flow behavior in the transition flow regime differs from that found in the continuum flow regime for the conditions investigated.

Having established a physical picture of the flowfield structure in a gap, the current study expands on the results presented in the previous analysis ${ }^{12}$ by investigating the effects of the $L / H$ ratio on the aerodynamic surface quantities. In this manner, the present investigation was undertaken in an attempt to assess the behavior of the heat transfer, pressure, and skin friction coefficients to changes on the gap $L / H$ ratio. The focus of the present study is the low-density region in the upper atmosphere, characterized by the transition flow regime, i.e., between the continuum flow and the free collision flow regime. In such a circumstance, the Direct Simulation Monte Carlo (DSMC) method will be employed to calculate the hypersonic twodimensional flow over the gaps.

\section{Computational Method and Procedure}

The choice of the numerical approach to be used in order to model rarefied non-equilibrium flows greatly relies on the extent of flow rarefaction. For near-continuum flows, it is usually sufficient to take into account the effects of rarefaction through the boundary conditions of slip velocity and temperature jump on the surface. Navier-Stokes equations, commonly used with these boundary conditions, can be derived from the Boltzmann equation under the assumption of small deviation of the distribution function from equilibrium. Nevertheless, Navier-Stokes equations became unsuitable for studying rarefied flows where the distribution function becomes considerable in non-equilibrium.

In order to study rarefied flow with a significant degree of non-equilibrium, the Direct Simulation Monte Carlo (DSMC) method ${ }^{13}$ is usually employed. The DSMC method has become the most common computational technique for modeling complex transitional flows of engineering interest. The DSMC method model a gas flow by using a computer to track the trajectory of simulated particles, where each simulated particle represents a fixed number of real gas particles. The simulated particles are allowed to move and collide, while the computer stores their position coordinates, velocities and other physical properties such as internal energy. The simulation is always calculated as unsteady flow. However, a steady flow solution is obtained as the large time state of the simulation.

The physical models employed in the present simulations are as follows. Intermolecular collisions are treated by using the variable hard sphere (VHS) molecular model, ${ }^{14}$ and the no time counter (NTC) collision sampling technique. ${ }^{15}$ The VHS model employs the simple hard sphere angular scattering law so that all directions are equally possible for post-collision velocity in the center-of-mass frame of reference. Nevertheless, the collision cross section depends on the relative speed of colliding molecules. Simulations are performed by using a non-reacting gas model, consisting of $76.3 \%$ of $\mathrm{N}_{2}$ and $23.7 \%$ of $\mathrm{O}_{2}$, while considering energy exchange between translational, rotational and vibrational modes. Energy partitioning is accounted for using the Borgnakke-Larsen statistical mode ${ }^{16}$ with rotational and vibrational collision numbers for internal relaxation, obtained in a collision energy-based procedure as suggested by Boyd ${ }^{17}$ for rotation and by Bird $^{18}$ for vibration. For a given collision, the probabilities are designated by the inverse of the relaxation numbers, which correspond to the number of collisions necessary, on average, for a molecule to relax.

\section{Geometry Definition}

The gap geometry considered in this work is the same as that presented in Paolicchi and Santos. ${ }^{12}$ It was assumed a flat plate with a gap of length $L$ and depth $H$. Figure 1 illustrates a schematic view of the model employed. The flat plate was selected by considering that the gap depth $H$ is much smaller than the nose radius $R$ of a reentry capsule, i.e., $H / R \ll 1$. Therefore, the hypersonic flow over the flat plate with a gap may be representative of the hypersonic flow over a gap located on the surface of a reentry vehicle.

Referring to Fig. 1, $M_{\infty}$ stands for the freestream Mach number, $H$ is the gap depth, $L$ is the gap length, $L_{u}$ the length of the gap upstream surface, and $L_{d}$ the length of the gap downstream surface. It was assumed a length $L$ of $3 \mathrm{~mm}$, and a depth $H$ of $3,6,9$, and $12 \mathrm{~mm}$. Therefore, the gaps investigated correspond to a length-to-depth ratio, $L / H$, of $1,1 / 2,1 / 3$ and $1 / 4$, respectively. In addition, $L_{u} / \lambda_{\infty}$ of 50 and $L_{d} / \lambda_{\infty}$ of 50 , where $\lambda_{\infty}$ is the freestream mean free path. It was considered that the flat plate is infinitely long but 


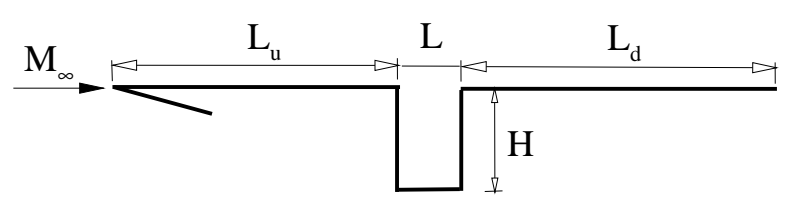

Figure 1. Drawing illustrating a schematic view of the gap configuration.

only the total length $L_{u}+L+L_{d}$ is investigated.

An understanding of the $L / H$ ratio effects on the aerodynamic surface properties can be gained by comparing the flowfield behavior of a flat plate with a gap to that without a gap. In this manner, a flat plate without a gap works as a benchmark for the cases with a gap, and will be referred herein as the flat-plate case.

\section{Freestream and Flow Conditions}

Freestream flow conditions used for the numerical simulations are those given by Paolicchi and Santos ${ }^{12}$ and summarized in Tab. 1. Based on this table, $U_{\infty}, T_{\infty}, p_{\infty}, \rho_{\infty}, \mu_{\infty}, n_{\infty}$, and $\lambda_{\infty}$ stand, respectively, for velocity, temperature, pressure, density, viscosity, number density, and molecular mean free path. In addition, the gas properties, such as mass fraction, $\chi$, molecular mass, $m$, molecular diameter, $d$, and viscosity index, $\omega$, associated with the working fluid, $\mathrm{N}_{2}$ and $\mathrm{O}_{2}$, are given by Bird ${ }^{13}$ and tabulated in Tab. 2 .

Table 1. Freestream flow conditions

\begin{tabular}{cccccccc}
\hline \hline Alt. $(\mathrm{km})$ & $U_{\infty}(\mathrm{m} / \mathrm{s})$ & $T_{\infty}(\mathrm{K})$ & $p_{\infty}\left(\mathrm{N} / \mathrm{m}^{2}\right)$ & $\rho_{\infty}\left(\mathrm{kg} / \mathrm{m}^{3}\right)$ & $\mu_{\infty}\left(\mathrm{Ns} / \mathrm{m}^{2}\right)$ & $n_{\infty}\left(\mathrm{m}^{-3}\right)$ & $\lambda_{\infty}(\mathrm{m})$ \\
\hline 70 & 7456 & 220.0 & 5.582 & $8.753 \times 10^{-5}$ & $1.455 \times 10^{-5}$ & $1.8209 \times 10^{21}$ & $9.03 \times 10^{-4}$ \\
\hline \hline
\end{tabular}

The freestream velocity $U_{\infty}$, assumed to be constant at $7456 \mathrm{~m} / \mathrm{s}$, corresponds to a freestream Mach number $M_{\infty}$ of 25 . The wall temperature $T_{w}$ is assumed constant at $880 \mathrm{~K}$. This temperature is chosen to be representative of the surface temperature near the stagnation point of a reentry capsule and is assumed to be uniform over the gap surface.

By assuming the gap depth $H$ as the characteristic length, the Knudsen number $K n_{H}$ corresponds to $0.3095,0.1548,0.1032$, and 0.0774 for depth $H$ of $3,6,9$, and $12 \mathrm{~mm}$, respectively. Finally, the Reynolds number $R e_{H}$ is around 121.7, 243.4, 365.1, and 486.8 for depth $H$ of $3,6,9$, and $12 \mathrm{~mm}$, respectively, also based on conditions in the undisturbed stream. 


\section{Computational Flow Domain and Grid}

For the numerical treatment of the problem, the flowfield around the gap is divided into five regions, which are subdivided into computational cells. The cells are further subdivided into subcells, two subcells/cell in each coordinate direction. The cell provides a convenient reference for the sampling of the macroscopic gas properties, while the collision partners are selected from the same subcell for the establishment of the collision rate. Dimensions of the cells must be such that changes in flow properties across each cell be small. In addition, linear dimensions of the cells should be small in comparison with the scale length of the macroscopic flow gradients normal to streamwise directions, which means that the cell dimensions should be the order of or even smaller than the local mean free path. ${ }^{19,20}$

The computational domain used for the calculation is made large enough so that gap disturbances do not reach the upstream and side boundaries, where freestream conditions are specified. A schematic view of the computational domain is illustrated in Fig. 2. According to this figure, side I-A is defined by the gap surface. Diffuse reflection with complete thermal accommodation is the condition applied to this side. Side I-B is a plane of symmetry, where all flow gradients normal to the plane are zero. At the molecular level, this plane is equivalent to a specular reflecting boundary. Sides II and III are the freestream sides

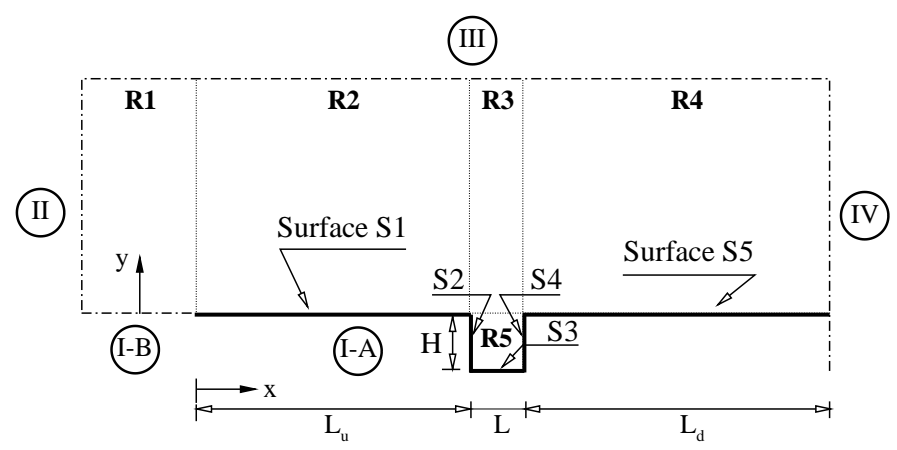

Figure 2. Drawing illustrating a schematic view of the computational domain. through which simulated molecules enter and exit. Side II is positioned at $5 \lambda_{\infty}$ upstream of the flat-plate leading edge, and side III defined at $30 \lambda_{\infty}$ above the flat plate. Finally, the flow at the downstream outflow boundary, side IV, is predominantly supersonic and vacuum condition is specified. ${ }^{13}$ At this boundary, simulated molecules can only exit. It should be mentioned in this context that, close to the wall, molecules may not be moving at supersonic speed. As a result, in this subsonic region close to the wall, there is an interaction between the flow and the downstream boundary. However, the extent of the upstream effect of this boundary condition can be determined by changing the length of the gap downstream surface. In doing so, the upstream disturbance is approximately of $6 \lambda_{\infty}$, as can be observed in the results presented in the subsequent sections.

Numerical accuracy in DSMC method depends on the grid resolution chosen as well as on the number of particles per computational cell. Moreover, the time step should be chosen to be sufficiently small in comparison with the local mean collision time. ${ }^{21,22}$ These effects were investigated to determine the number of cells and the number of particles required to achieve grid independence solutions. A grid independence study was made with three different structured meshes - coarse, standard and fine - in each coordinate direction. The effect of altering the cell size in the $x$ - and $y$-directions was investigated for a coarse and fine

Table 3. Number of cells in the (x-direction) and [y-direction] investigation for the $L / H=1$ case.

\begin{tabular}{lcccccc}
\hline \hline & Region 1 & Region 2 & Region 3 & Region 4 & Region 5 & Total number of cells \\
\hline Coarse & $(10 \times 80)$ & $(65 \times 110)$ & $(10 \times 110)$ & $(65 \times 120)$ & $(20 \times 40)$ & 17,650 \\
& {$[20 \times 40]$} & {$[130 \times 55]$} & {$[20 \times 55]$} & {$[130 \times 60]$} & {$[40 \times 20]$} & 17,650 \\
Standard & $20 \times 80$ & $130 \times 110$ & $20 \times 110$ & $130 \times 120$ & $40 \times 40$ & 35,300 \\
Fine & $(40 \times 80)$ & $(260 \times 110)$ & $(40 \times 110)$ & $(260 \times 120)$ & $(80 \times 40)$ & 70,600 \\
& {$[20 \times 160]$} & {$[130 \times 220]$} & {$[20 \times 220]$} & {$[130 \times 240]$} & {$[40 \times 80]$} & 70,600 \\
\hline \hline
\end{tabular}


grids with, respectively, $50 \%$ less and $100 \%$ more cells with respect to the standard grid. In addition, each grid was made up of non-uniform cell spacing in both directions. Moreover, point clustering is used close to solid walls and to the horizontal plane connecting the two corners. Table 3 tabulates the number of cells employed in the five regions (from R1 to R5 in Fig. 2) for coarse, standard, and fine grids for the $L / H=1$ case.

The effect (not shown) of changing the cell size in both directions on the heat transfer, pressure and skin friction coefficients was rather insensitive to the range of cell spacing considered, indicating that the standard grid, with a total of 35,300 cells, for the $L / H=1$ case, is essentially grid independent.

A similar examination was made for the number of molecules. The standard grid for the $L / H=1$ case corresponds to, on average, a total of 741,200 molecules. Two new cases using the same grid were investigated. These two new cases correspond to 370,600 and 1482,400 molecules in the entire computational domain. As the three cases presented the same results (not shown) for the heat transfer, pressure and skin friction coefficients, hence the standard grid with a total of 741,200 molecules is considered enough for the computation of the flowfield properties. A discussion of the effects of the cell size, time step, and number of molecules variations on the aerodynamic surface quantities for the gaps presented herein is described in detail by Paolicchi. ${ }^{23}$

\section{Computational Results and Discussion}

This section focuses on the effects that take place in the aerodynamic surface quantities due to variations on the gap $L / H$ ratio. Aerodynamic surface quantities of particular interest in the transitional flow regime are number flux, heat flux, wall pressure, and shear stress. In this scenario, this section discusses and compares differences of these quantities expressed in a coefficient form.

Before proceeding with the analysis of the aerodynamic surface quantities, it proves instructive to first present the flow topology inside the gaps. In doing so, tangential velocity profiles inside the gaps are demonstrated in Fig. 3 for three sections parameterized by the $L / H$ ratio. In this set of plots, the tangential velocity $u$ is normalized by the freestream velocity $U_{\infty}$, the dimensionless height $Y$ corresponds to the height $y$ normalized by the freestream mean free path $\lambda_{\infty}$, and $X_{L}^{\prime}$ represents the distance $\left(x-L_{u}-L / 2\right)$ normalized by the gap length $L$. According to this set of plots, the behavior of the tangential velocity profiles is similar for the sections shown. The tangential velocity ratio is zero at the bottom of the gaps, it becomes negative for the range $-7<Y<-1$, and then it is positive for $Y>-1$.

Normal velocity profiles inside the gaps are displayed in Fig. 4 for three sections as a function of the

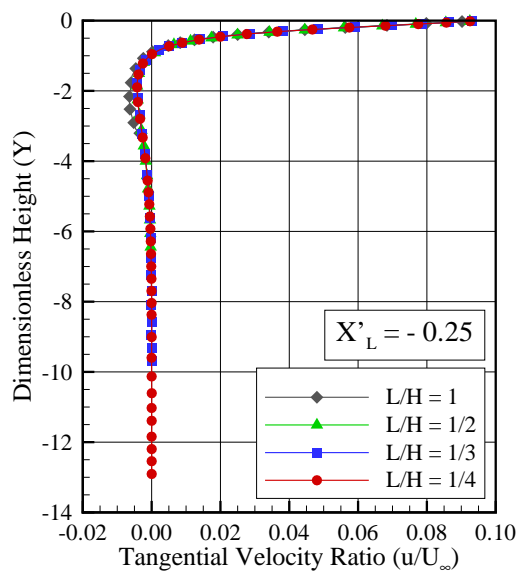

(a)

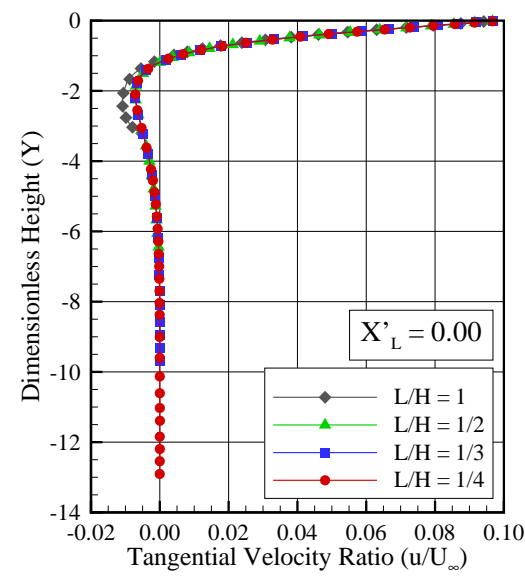

(b)

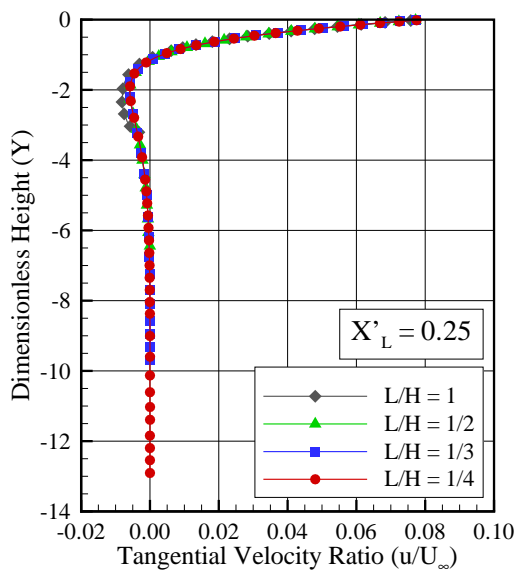

(c)

Figure 3. Distribution of tangential velocity $\left(u / U_{\infty}\right)$ inside the gaps for three longitudinal sections defined by $X_{L}^{\prime}$ of (a) -0.25 , (b) 0.0 , and (c) 0.25 . 


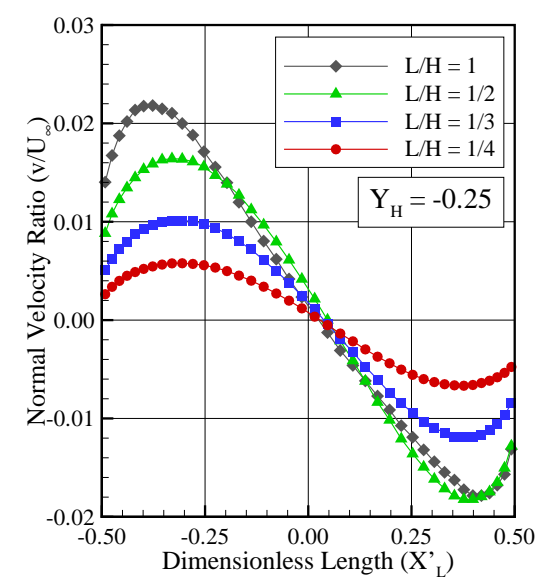

(a)

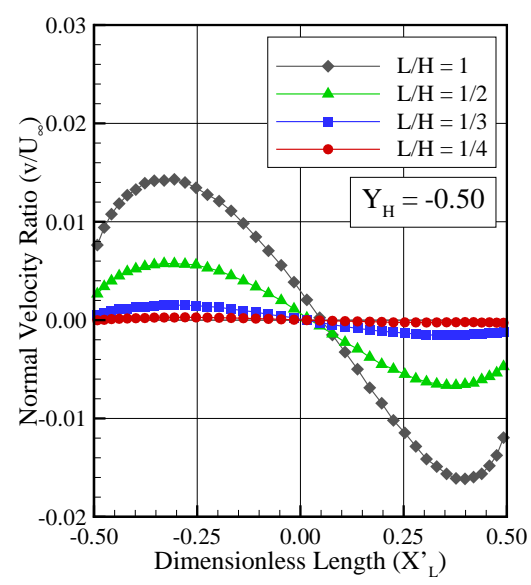

(b)

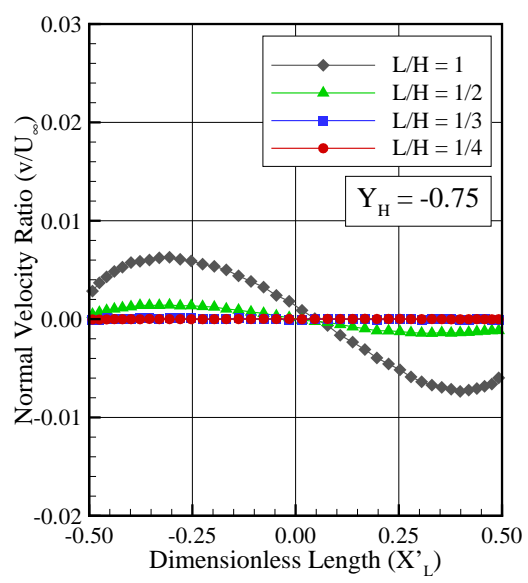

(c)

Figure 4. Distribution of normal velocity $\left(v / U_{\infty}\right)$ inside the gaps for three transversal sections defined by $Y_{H}$ of (a) $-0.25,(b)-0.50$, and (c) -0.75 .

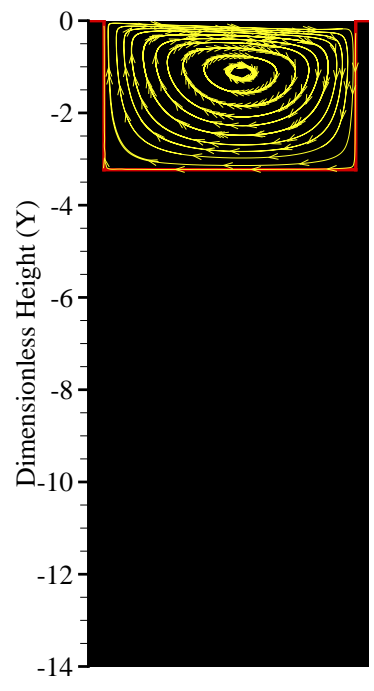

(a) $L / H=1$

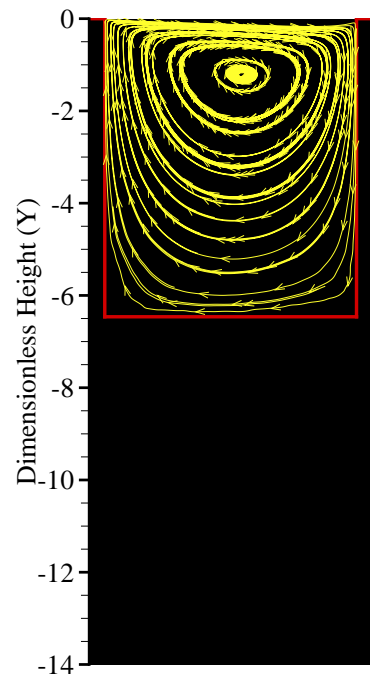

(b) $L / H=1 / 2$

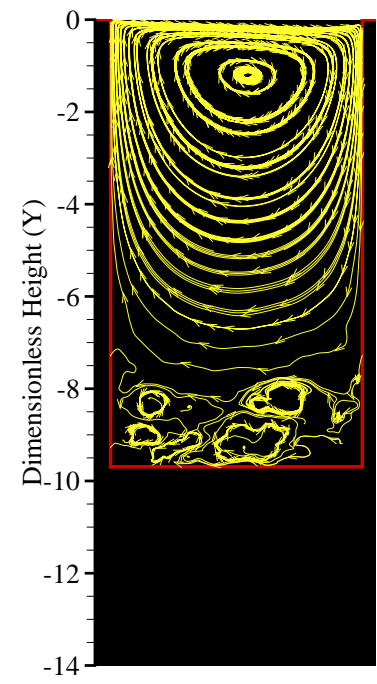

(c) $L / H=1 / 3$

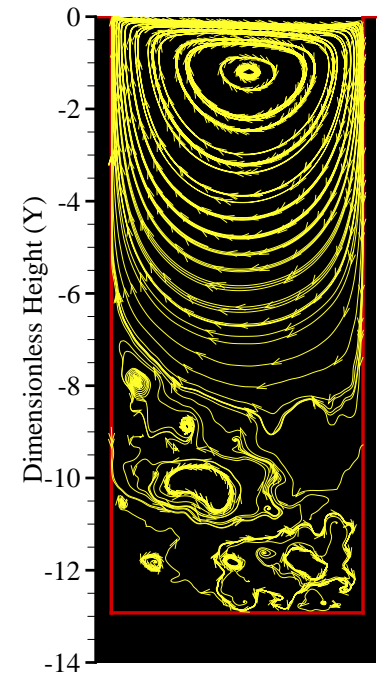

(d) $L / H=1 / 4$

Figure 5. Distribution of streamline traces for $L / H$ ratio of (a) 1, (b) 1/2, (c) 1/3 and (d) 1/4.

streamwise distance and parameterized by the $L / H$ ratio. In this set of diagrams, the normal velocity $v$ is normalized by the freestream velocity $U_{\infty}$, and $Y_{H}$ corresponds to the transversal sections $y$ normalized by the gap depth $H$. According to these plots, near to the gap backward face, $-0.5<X_{L}^{\prime}<0.0$, the normal velocity ratio profiles present positive values, meaning that the flow is moving upward. Conversely, at the vicinity of the gap forward face, $0.0<X_{L}^{\prime}<0.5$, the normal velocity ratio profiles present negative values, indicating that the flow is moving downward. Therefore, based on these two opposite behaviors for the normal velocity ratio, it may be inferred in passing that there is a region of a clockwise circulating flow. In addition, of particular interest is the behavior of the normal velocity profiles for the $L / H<1 / 2$ cases 
near to the bottom surface of the gaps, i.e., for section $Y_{H}=-0.75$. It is quite apparent that the $v$-velocity component is zero, indicating that there are no recirculation regions at the vicinity of the gap floor for these cases.

At this point, it is worth taking a closer look at the recirculation region. In this manner, streamline traces inside the gaps are depicted in Fig. 5 as a function of the $L / H$ ratio. Based on Fig. 5 , it is clearly noticed that the flow within the gaps is characterized by a primary vortex system. For the $L / H=1$ and $1 / 2$ cases, it is noteworthy that the recirculation region fills the entire gaps. Conversely, for the $L / H=1 / 3$ and $1 / 4$ cases, the recirculation regions do not fill the entire gaps. In addition, the external stream does not reattach at the bottom surface of the gaps.

Still referring to Fig. 5, it is clearly noticed that the gap flow topology observed here in a rarefied environment differs from that usually observed in the continuum flow regime. In the continuum flow regime, the gap flow topology is defined by the development of a column of counter-rotating vortices within the gap caused by the main stream flow, where the number of vortices is approximately given by $H / L$. In addition, alternating hot spots are developed in the gap when the vortices directionally align and impinge on the gap sidewall. $^{24}$ Of particular interest in Fig. 5 is the flow behavior for $L / H=1 / 3$ and $1 / 4$, where the flow exhibits an irregular or chaotic structural motion in the second half part of the gaps, more precisely for $Y<-7$.

\section{A. Number Flux}

The number flux, $N$, is calculated by sampling the molecules impinging on the surface by unit time and unit area. Effects of the $L / H$ ratio on the number flux are illustrated in Figs. 6 and 7. In this group of plots, $N_{f}$ represents the number flux $N$ normalized by $n_{\infty} U_{\infty}$, where $n_{\infty}$ and $U_{\infty}$ correspond, respectively, to the freestream number density and freestream velocity. Also, $X$ stands for the length $x$ normalized by the freestream mean free path $\lambda_{\infty}, X^{\prime}$ refers to the length $\left(x-L_{u}\right)$ normalized by the gap length $L$, and $Y_{H}^{\prime}$ refers to the height $y$ normalized by the gap depth $H$. Also, S1, S3, and S5 correspond to gap horizontal surfaces, i.e., the upstream, bottom, and downstream gap surfaces, S2 and S4 refer to the vertical gap surfaces, i.e., the backward and the forward faces. As a basis of comparison, the dimensionless number flux $N_{f}$ for the flat-plate case, i.e., a flat plate without a gap, is also illustrated in Fig. 6.

According to Fig. 6, it is clearly seen that along surface $\mathrm{S} 1$ the number flux behavior follows that presented by the flat-plate case for the $L / H$ ratio investigated in this work, since no upstream disturb is caused by the gap presence. Along surface S5, no appreciable changes are observed in the number flux distribution. It is seen that $N_{f}$ basically follows the same pattern of that presented by the flat-plate case. Nevertheless,

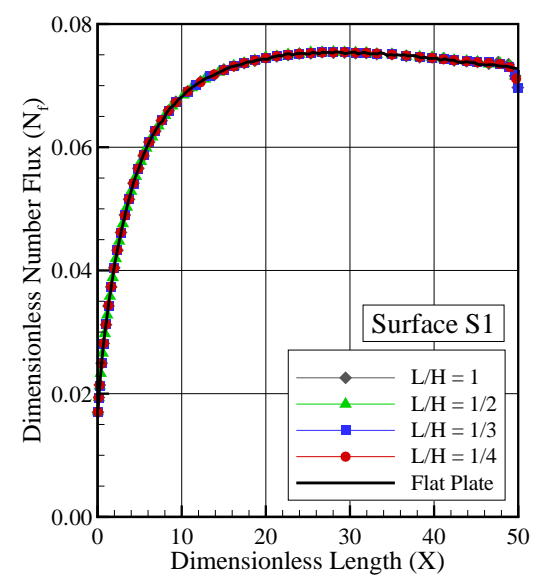

(a)

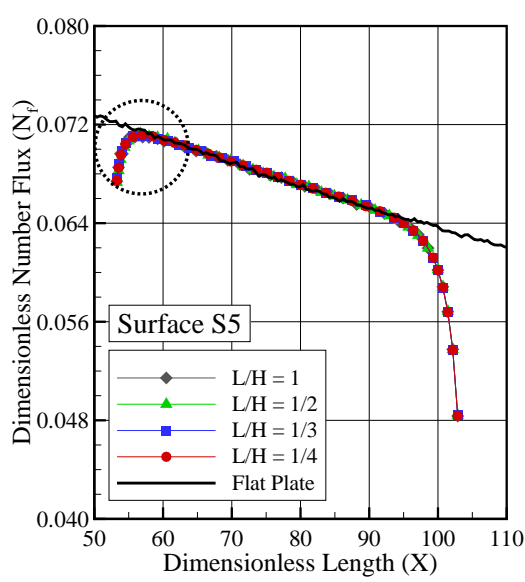

(b)

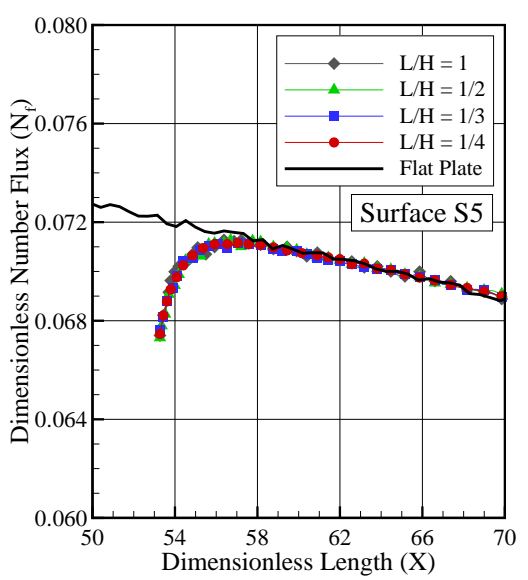

(c)

Figure 6. Dimensionless number flux $N_{f}$ distribution along gap surfaces (a) S1 and (b) S5, along with a (c) magnified view of the curves at the vicinity of the gap shoulder. 


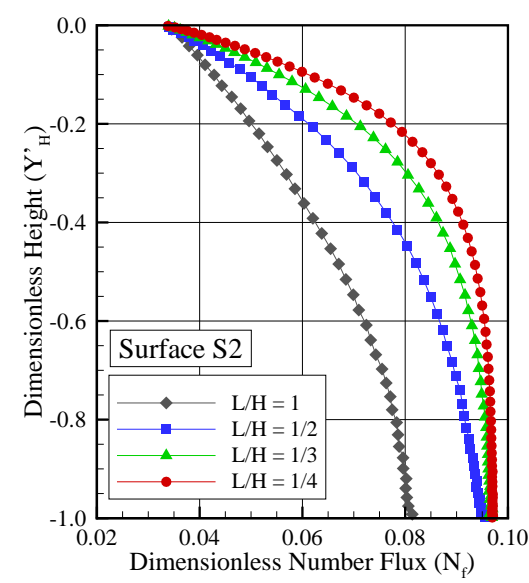

(a) Backward surface

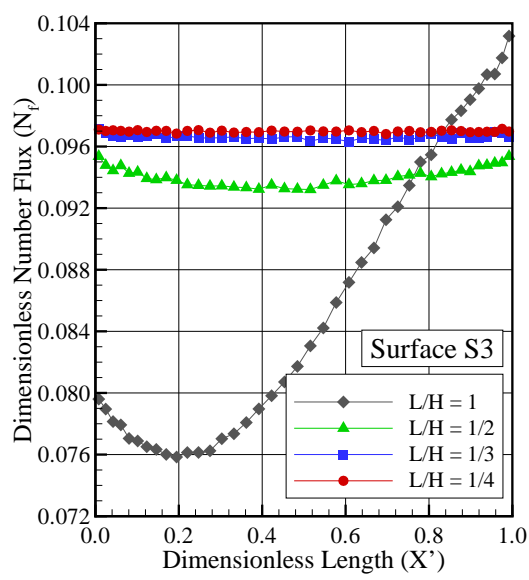

(b) Bottom surface

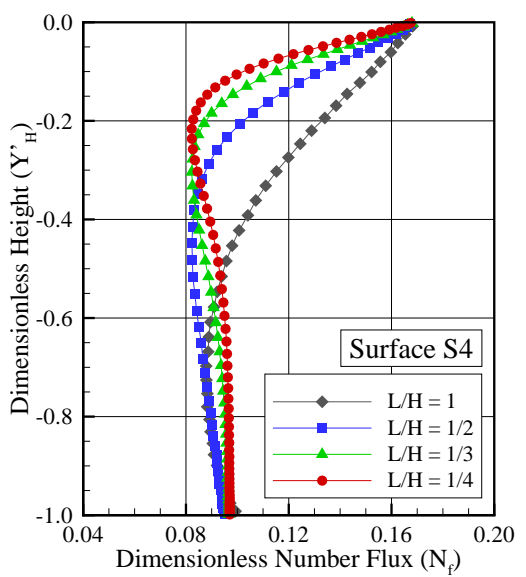

(c) Forward surface

Figure 7. Dimensionless number flux $N_{f}$ distribution along gap surfaces (a) S2, (b) S3, and (c) S4.

attention should be paid to two interesting features. At the beginning of surface S5, the number flux is lower than that for the flat-plate case. Due to the flow expansion on the gap shoulder, density is low at the beginning of surface S5. ${ }^{12}$ As a result, the number flux is low compared to that for the flat-plate case. A magnified view of this behavior is shown in detail in Fig. 6(c). In addition, a dramatically reduction in the number flux is observed at the end of the surface S5. The reason for that is associated with the vacuum condition assumed for the downstream outflow boundary, side IV, as explained earlier.

Referring to Fig. 7, for the backward face, surface S2, the dimensionless number flux $N_{f}$ is low at the top of the gap and increases gradually along the surface up to the corner at the bottom surface. Along the gap floor, surface $\mathrm{S} 3$, the number flux behavior also relies on $L / H$ ratio. It is seen that it increases with decreasing the $L / H$ ratio. Nevertheless, it seems to reach a constant value for $L / H<1 / 3$. In the following, along the forward face, surface S4, $N_{f}$ basically increases from the corner, at the bottom surface, up to the top of the gap. This behavior is in contrast to that observed for the backward face. In fact, this is an expected behavior in the sense that it is directly related to the flow recirculation inside the gap. Due to the clockwise flow recirculation, at the vicinity of the surface S4, density is higher than that close to the surface S2, as shown by Paolicchi and Santos. ${ }^{12}$ Therefore, a large flux of molecules colliding to this surface is expected.

\section{B. Heat Transfer Coefficient}

The heat transfer coefficient $C_{h}$ is defined as follows,

$$
C_{h}=\frac{q_{w}}{\frac{1}{2} \rho_{\infty} U_{\infty}^{3}}
$$

where the heat flux $q_{w}$ to the body surface is calculated by the net energy flux of the molecules impinging on the surface. A flux is regarded as positive if it is directed toward the body surface. The net heat flux $q_{w}$ is related to the sum of the translational, rotational and vibrational energies of both incident and reflected molecules as defined by,

$$
q_{w}=q_{i}-q_{r}=\frac{F_{N}}{A \Delta t}\left\{\sum_{j=1}^{N}\left[\frac{1}{2} m_{j} c_{j}^{2}+e_{R j}+e_{V j}\right]_{i}-\sum_{j=1}^{N}\left[\frac{1}{2} m_{j} c_{j}^{2}+e_{R j}+e_{V j}\right]_{r}\right\}
$$

where $F_{N}$ is the number of real molecules represented by a single simulated molecule, $\Delta t$ is the time step, $A$ the area, $N$ is the number of molecules colliding with the surface by unit time and unit area, $m$ is the mass 


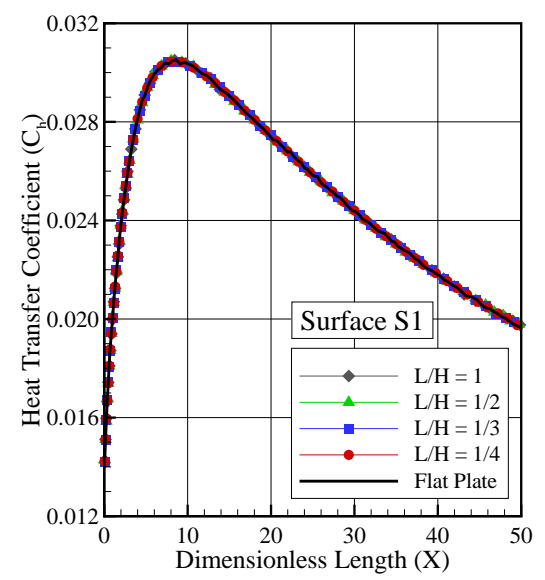

(a)

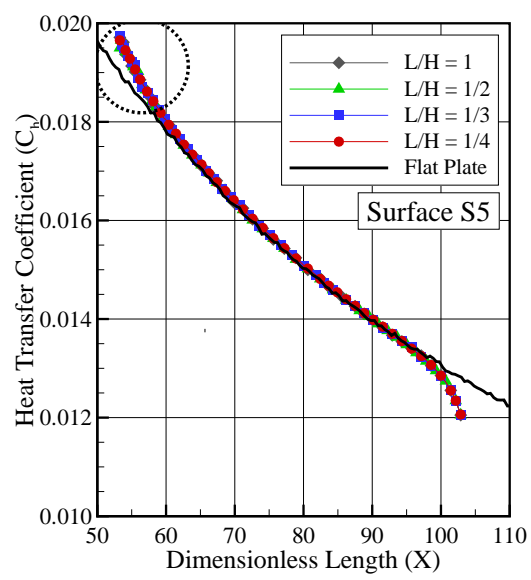

(b)

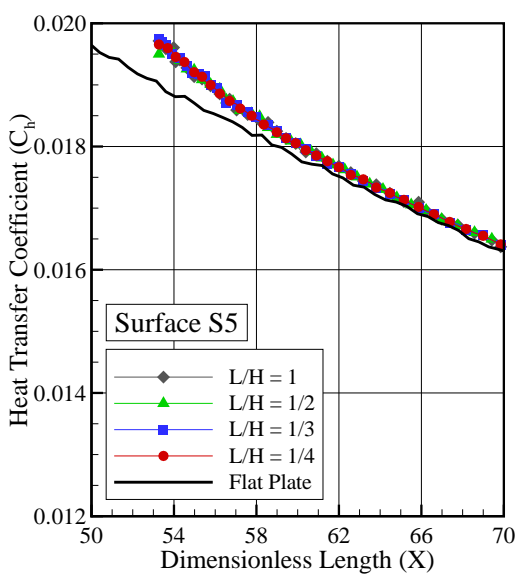

(c)

Figure 8. Heat transfer coefficient $C_{h}$ distribution along gap surfaces (a) S1 and (b) S5, along with a (c) magnified view of the curves at the vicinity of the gap shoulder.

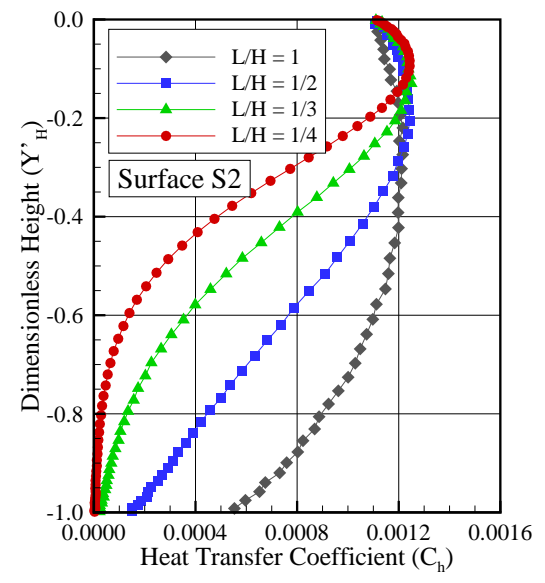

(a) Backward surface

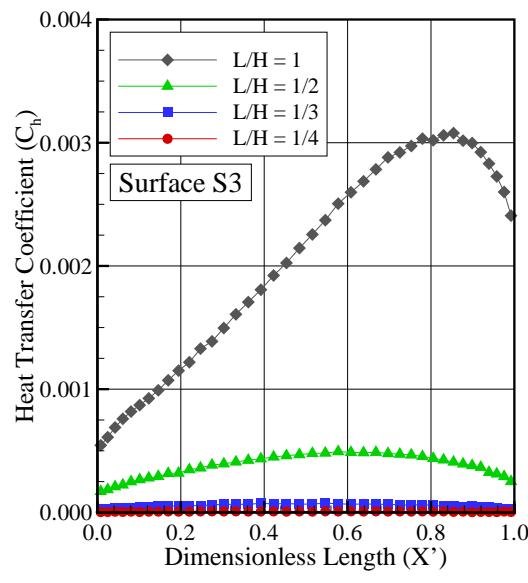

(b) Bottom surface

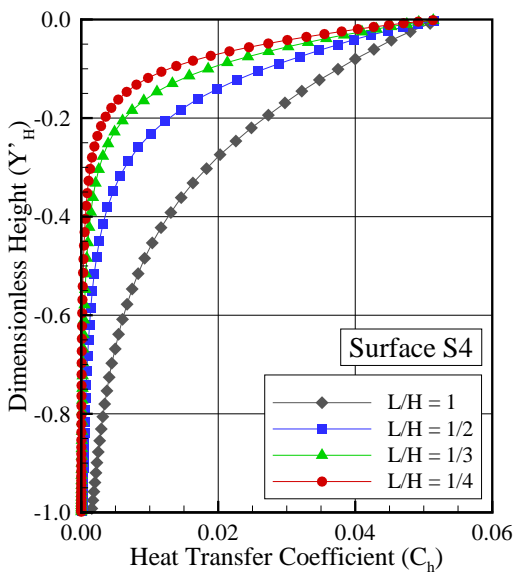

(c) Forward surface

Figure 9. Heat transfer coefficient $C_{h}$ distribution along gap surfaces (a) S2, (b) S3, and (c) S4.

of the molecules, $c$ is the velocity of the molecules, $e_{R}$ and $e_{V}$ stand for rotational and vibrational energies, respectively. Subscripts $i$ and $r$ refer to incident and reflect molecules.

The dependence of the heat transfer coefficient $C_{h}$ on the $L / H$ ratio is demonstrated in Fig. 8 for surfaces S1 and S5, and in Fig. 9 for surfaces S2, S3, and S4. According to Fig. 8, no appreciable changes are observed in the heat transfer coefficient $C_{h}$ along surface $\mathrm{S} 1$ due to changes in the $L / H$ ratio. Along surface $\mathrm{S} 1$, it is observed that the heat transfer coefficient for the gaps is the same of that presented by the flat-plate case. $C_{h}$ is low at the leading edge, increases up to a peak value, $C_{h}=0.0305$, around section $X=8.44$, and decreases downstream along the plate. Along surface S5, it is seen that the heat transfer coefficient $C_{h}$ is larger than that for the flat-plate case at the vicinity of the gap corner, defined by the surface-S4/surface-S5 junction. Nonetheless, as the flow moves downstream along the surface, the heat transfer coefficient $C_{h}$ basically recovers the value obtained for the flat-plate case. This behavior is illustrated in the magnified 
view presented in Fig. 9(c).

Referring to Fig. 9, for the backward face, surface S2, the heat transfer coefficient $C_{h}$ is high at the top of the gap and increases to a maximum value close to the shoulder, surface-S1/surface-S2 junction. Afterwards, $C_{h}$ drops off along the surface up to the corner at the bottom surface. Along the gap floor, surface S3, the heat transfer coefficient depends on the $L / H$ ratio. It is noted that the behavior for the $L / H=1$ case differs from those cases defined by $L / H<1$. While $C_{h}$ increases up to a peak value close to the surface-S3/surface-S4 junction for the $L / H=1$ case, $C_{h}$ practically goes to zero for the cases defined by $L / H<1$. It should be remarked in this context that, for $L / H<1$ cases, the recirculation does not reach the bottom of the gaps. Also, density is higher in this region. As a result, the molecular collision rate is larger compared to that for other regions, the flow comes to the thermal equilibrium, and the temperature reaches the wall temperature, as shown by Paolicchi and Santos. ${ }^{12}$

Still referring to Fig. 9, for the forward face, surface S4, the heat transfer coefficient $C_{h}$ increases monotonically from basically zero, at the surface-S3/surface-S4 junction, to the maximum value, $C_{h} \approx 0.051$, at the surface-S4/surface-S5 junction. After that, $C_{h}$ reaches the heat transfer coefficient for the flat-plate case along surface S5. It is very encouraging to observe that the heat transfer coefficient $C_{h}$ to the forward face is roughly one order of magnitude larger than that to the bottom surface, and this is one order of magnitude larger than that to the backward surface. This is explained by the fact that, at the vicinity of the backward face, the flow experiences an expansion. In contrast, at the vicinity of the forward face, the flow experiences a compression due to the recirculation region inside the gaps.

Before proceeding with the analysis, it is desirable to take a closer look at these results. In doing so, the peak values for the heat transfer coefficient $C_{h}$, approximately 0.051 for the $L / H$ ratio of $1,1 / 2,1 / 3$, and $1 / 4$, at the gap forward corner, are compared to that predicted for a smooth surface, i.e., a flat plate without a gap. Based on Fig. 8, the maximum value for $C_{h}$ is around to 0.0305 at a station $8.44 \lambda_{\infty}$ from the leading edge. Therefore, the peak values of $C_{h}$ for the gaps are approximately 1.67 times that for a smooth surface.

\section{Pressure Coefficient}

The pressure coefficient $C_{p}$ is defined as follows,

$$
C_{p}=\frac{p_{w}-p_{\infty}}{\frac{1}{2} \rho_{\infty} U_{\infty}^{2}}
$$

where the pressure $p_{w}$ on the body surface is calculated by the sum of the normal momentum fluxes of both incident and reflected molecules at each time step as follows,

$$
p_{w}=p_{i}-p_{r}=\frac{F_{N}}{A \Delta t} \sum_{j=1}^{N}\left\{\left[(m v)_{j}\right]_{i}-\left[(m v)_{j}\right]_{r}\right\}
$$

where $v$ is the velocity component of the molecule $j$ in the surface normal direction.

The impact on the pressure coefficient $C_{p}$ due to changes in the gap $L / H$ ratio is displayed in Figs. 10 and 11. Referring to Fig. 10, it is observed that the pressure coefficient follows the same trend as that presented for the dimensionless number flux in the sense that, along surface $S 1$, the pressure coefficient presents the same behavior for the flat-plate case. Along surface S5, it is seen that the pressure coefficient $C_{p}$ behavior is similar to that for the flat-plate case, except at the vicinity of the gap forward corner, i.e., the surface-S4/surface-S5 junction. Near to this corner, a significant reduction in the pressure coefficient is observed when compared to the pressure coefficient of the flat-plate case. The reason for that is due to the flow expansion around this corner. Moreover, this pressure decrease is associated to the significant reduction in the number flux, as shown in Figs. 6 . This behavior in the pressure coefficient is shown in detail in the magnified view in Fig. 10(c).

According to Fig. 11, it is seen that the pressure coefficient $C_{p}$ basically follows a similar behavior as that presented by the number flux in the sense that, for the backward face, surface S2, it is low at the shoulder, $Y_{H}^{\prime}=0$, and increases downward along the surface, reaching the maximum value at the bottom, station

$Y_{H}^{\prime}=-1.0$. In what follows, for the gap floor, surface S3, the pressure coefficient distribution relies on the gap $L / H$ ratio. For the $L / H=1$ case, $C_{p}$ increases along the surface, and reaches the maximum value at the vicinity of the surface-S3/surface-S4 junction. Nevertheless, for $L / H<1$ cases, the pressure coefficient 


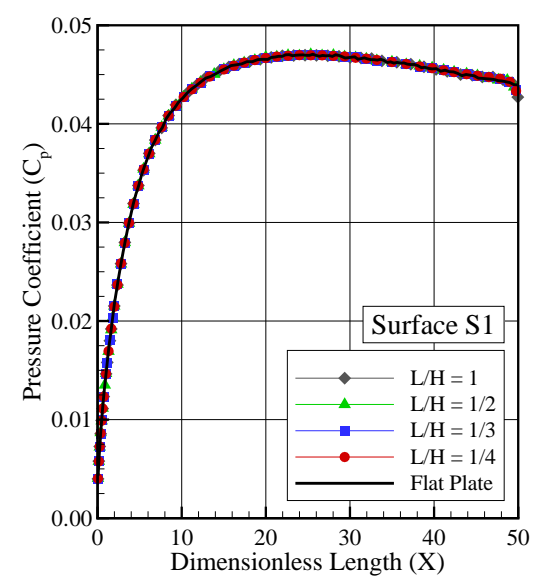

(a)

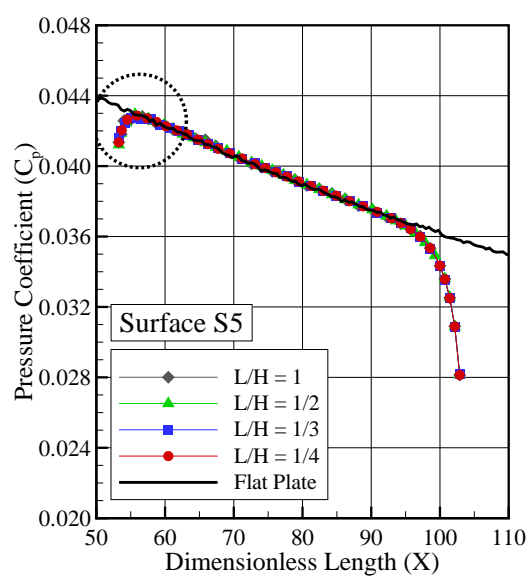

(b)

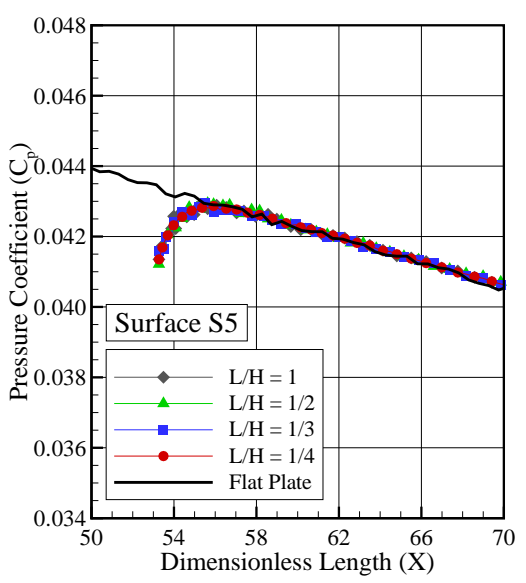

(c)

Figure 10. Pressure coefficient $C_{p}$ distribution along gap surfaces (a) S1 and (b) S5, along with a (c) magnified view of the curves at the vicinity of the gap shoulder.

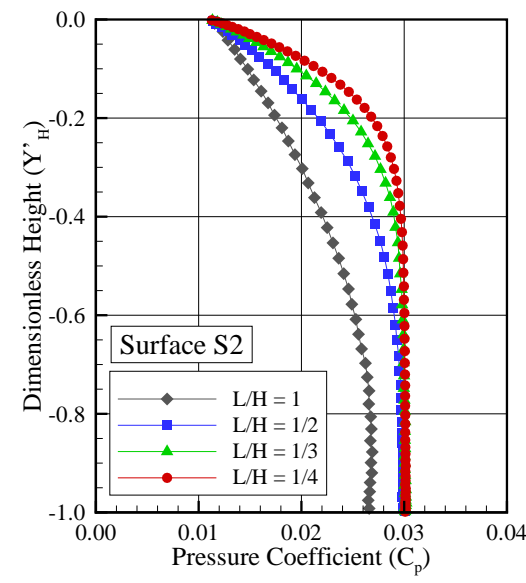

(a) Backward surface

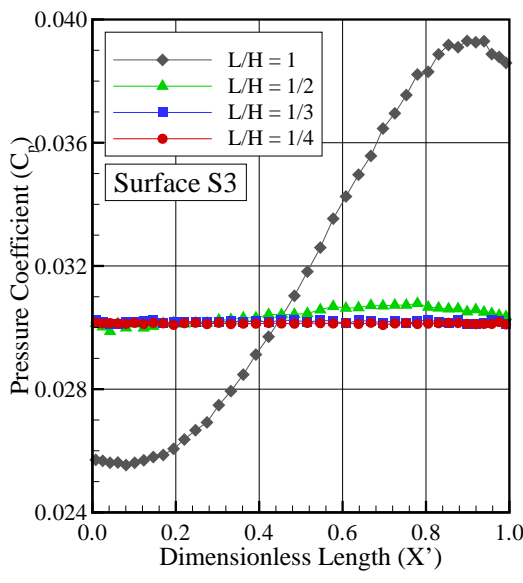

(b) Bottom surface

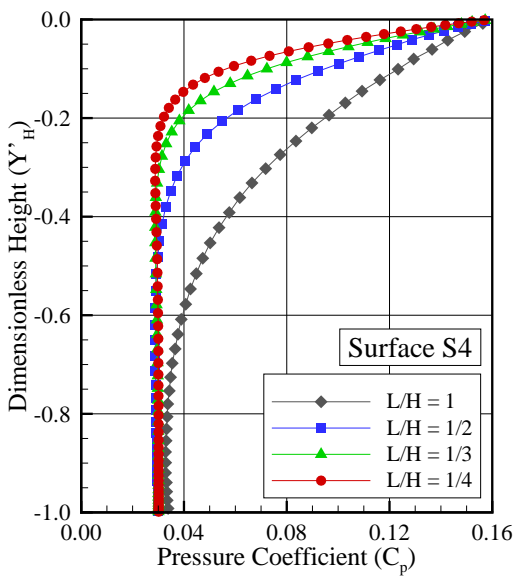

(c) Forward surface

Figure 11. Pressure coefficient $C_{p}$ distribution along gap surfaces (a) S2, (b) S3, and (c) S4.

is constant along the surface. Finally, along the forward face, surface S4, the pressure coefficient behavior is in contrast to that observed along surface $\mathrm{S} 2$ in the sense that $C_{p}$ presents the lower value at the station $Y_{H}^{\prime}=-1.0$, and increases monotonically upward along the surface, reaching the peak value at the shoulder, $Y_{H}^{\prime}=0$. It may be inferred in passing that this is an expected behavior since the flow within the gap is characterized by the appearance of a recirculation region. As shown in Fig. 5, the streamline pattern shows that the flow is characterized by a primary vortex system for $L / H$ ratio investigated, where a clockwise recirculation structure fills the entire gap for the $L / H=1$ case. Conversely, for the $L / H<1$ cases, the recirculation region does not reach the gap floor, surface S3.

For the time being, it proves instructive to compare the maximum values for the pressure coefficient observed in the gaps with that of a smooth surface, i.e., a flat plate without a gap. For comparison purpose, the peak value for $C_{p}$ is around 0.157 observed at the gap forward corner, surface-S4/surface-S5 junction. 
Based on Fig. 10, for the flat-plate case, the maximum value for $C_{p}$ is around to 0.047 at a station $22.8 \lambda_{\infty}$ from the leading edge. Therefore, the $C_{p}$ peak value for the gaps is around 3.34 times larger than the peak value for a smooth surface.

\section{Skin Friction Coefficient}

The skin friction coefficient $C_{f}$ is defined as follows,

$$
C_{f}=\frac{\tau_{w}}{\frac{1}{2} \rho_{\infty} U_{\infty}^{2}}
$$

where the shear stress $\tau_{w}$ on the body surface is calculated by the sum of the tangential momentum fluxes of both incident and reflected molecules impinging on the surface at each time step by the following expression,

$$
\tau_{w}=\tau_{i}-\tau_{r}=\frac{F_{N}}{A \Delta t} \sum_{j=1}^{N}\left\{\left[(m u)_{j}\right]_{i}-\left[(m u)_{j}\right]_{r}\right\}
$$

where $u$ is the velocity component of the molecule $j$ in the surface tangential direction.

It is worthwhile to note that for the special case of diffuse reflection, the gas-surface interaction model adopted in the present work, the reflected molecules have a tangential moment equal to zero, since the molecules essentially lose, on average, their tangential velocity components. In this fashion, the contribution of $\tau_{r}$ in Eq. 6 is equal to zero.

The effect of the $L / H$ ratio on the skin friction coefficient $C_{f}$ is depicted in Fig. 12 for surfaces $\mathrm{S} 1$ and S5, and in Fig. 13 for surfaces S2, S3, and S4. According to Fig. 12, it is noted that the skin friction coefficient $C_{f}$ follows a similar behavior of that presented by the heat transfer coefficient $C_{h}$. Along surface $\mathrm{S} 1$, it is seen that $C_{f}$ reproduces the skin friction distribution for the flat-plate case. Along surface S5, similar to the heat transfer coefficient, it is noticed that the skin friction coefficient $C_{f}$ is larger than that for the flat-plate case, especially in the vicinity of the gap downstream corner, defined by the surface-S4/surface-S5 junction. This slight increase in $C_{f}$ is illustrated in a magnified view in Fig. 12(c). Nevertheless, as the flow moves downstream along surface S5, the skin friction coefficient $C_{f}$ reaches the value observed for the flat-plate case. Finally, it should be mentioned that the peak values for the skin friction coefficient along surfaces S1

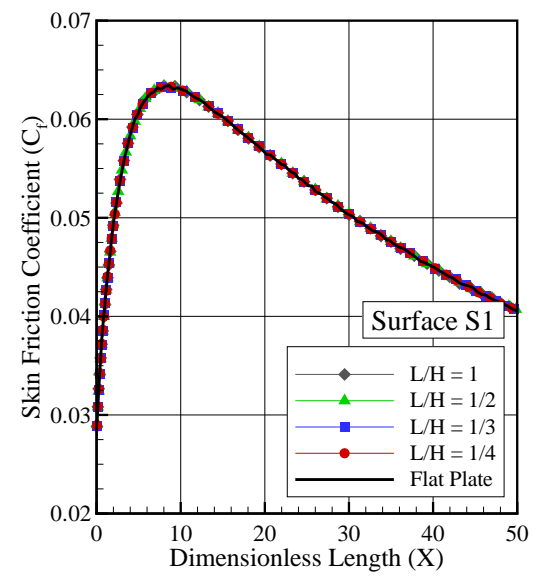

(a)

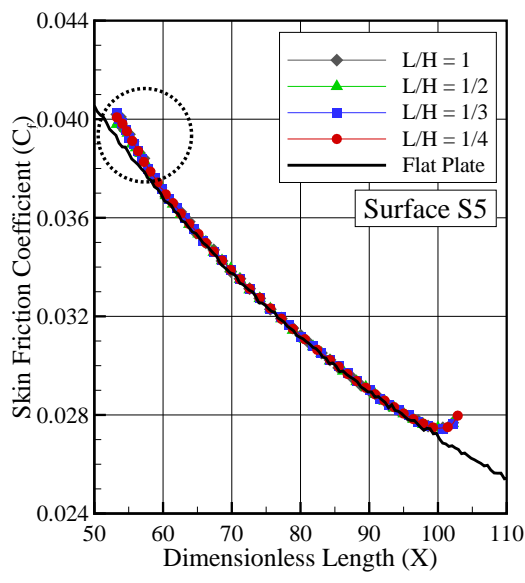

(b)

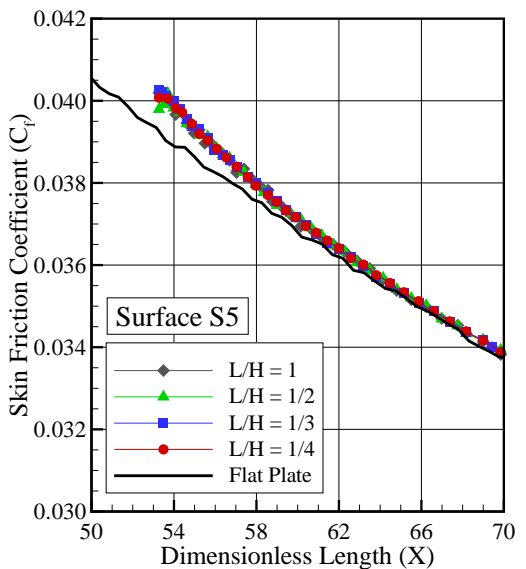

(c)

Figure 12. Skin friction coefficient $C_{f}$ distribution along gap surfaces (a) S1 and (b) S5, along with a (c) magnified view of the curves at the vicinity of the gap shoulder. 


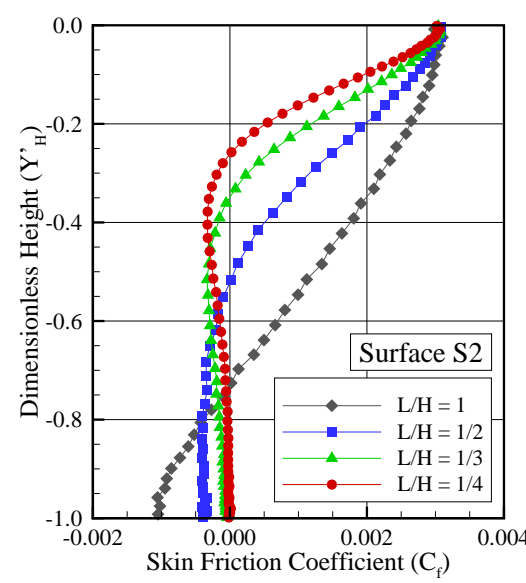

(a) Backward surface

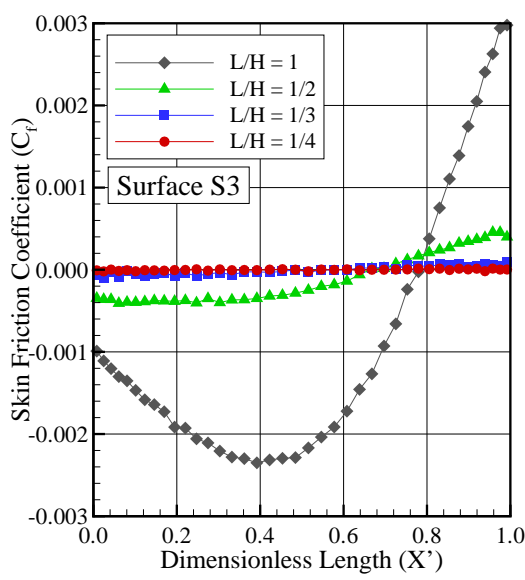

(b) Bottom surface

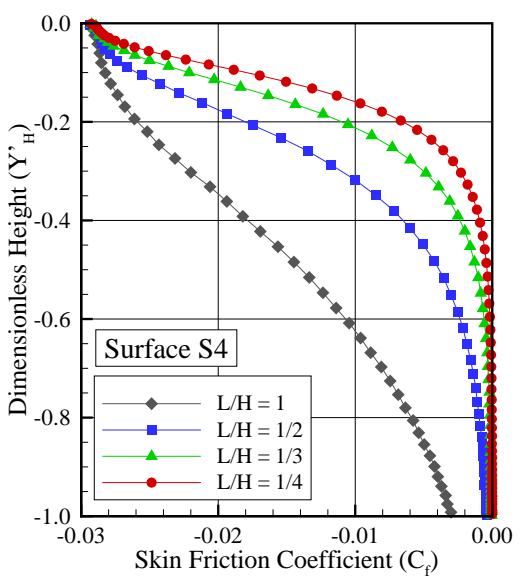

(c) Forward surface

Figure 13. Skin friction coefficient $C_{f}$ distribution along gap surfaces (a) S2, (b) S3, and (c) S4.

and S5 are larger than those observed for the pressure coefficient. As a result, tangential forces, associated to the shear stress, are larger than normal forces, related to the wall pressure.

Looking to Fig. 13, for the backward face, surface S2, the skin friction coefficient in general presents the peak value at the shoulder, $Y_{H}^{\prime}=0$, decreases along the upper half part of the surface, and basically reaches a negative constant value along the lower half part of the surface. In what follows, for the gap floor, surface S3, the skin friction coefficient is negative near the vicinity of the surface-S2/surface-S3 junction, and becomes positive at the vicinity of the surface-S3/surface-S4 junction. Nevertheless, as the $L / H$ ratio increases, $C_{f} \approx 0$ is observed along the entire surface. Finally, along the forward face, surface $\mathrm{S} 4$, the skin friction coefficient starts from zero at the vicinity of the surface-S3/surface-S4 junction and decreases negatively up to the minimum value at the shoulder, $Y_{H}^{\prime}=0$.

Usually, as $C_{f}$ changes from positive to negative value, the condition $C_{f}=0$ may indicate the presence of a backflow, an attachment or reattachment point in the 2-D flow. In the present account, these changes are directly related to the clockwise recirculation region inside the gaps. For the $L / H$ ratio investigated, the flowfield structure inside the gaps was defined by only one vortex system, in contrast to the gap flowfield structure usually observed in the continuum flow regime. ${ }^{24}$

\section{Concluding Remarks}

Computations of a rarefied hypersonic flow on a family of gaps have been performed by using the Direct Simulation Monte Carlo (DSMC) method. The calculations provided information concerning the nature of the aerodynamic surface quantities on the gaps. Effects of the length-to-depth ratio on the number flux, heat transfer, pressure and skin friction coefficients for a representative range of parameters were investigated. The length-to-depth ratio ranged from 1 to 1/4, which corresponded Knudsen numbers in the transitional flow regime.

On the basis of the foregoing results it was concluded that, for the range of the conditions investigated, the aerodynamic quantities acting on the gap surface depend on the $L / H$ ratio. It was found that the pressure load and the heat load presented the peak values at the corner of the gap forward face. The analysis showed that these loads are much larger than those attained in a smooth surface, represented by a flat plate without a gap. 


\section{Acknowledgments}

The authors would like to thank the financial support for this research, provided by CNPq (Conselho Nacional de Desenvolvimento Científico e Tecnológico) under Grant No. 473267/2008-0, and the financial support in order to attend this conference, provided by Capes (Coordenação de Aperfeiçoamento de Pessoal de Nível Superior) under Grant No. 2848/13-9.

\section{References}

${ }^{1}$ Petley, D. H., Smith, D. M., Edwards, C. L. W., Carlson, A. B., and Hamilton II, H. H., "Surface Step Induced Gap Heating in the Shuttle Thermal Protection System", Journal of Spacecraft and Rockets, Vol. 11, No. 2, 1984, pp. $156-161$.

${ }^{2}$ Pitts, W. C., and Murbach, M. S., "Flight Measurements of Tile Gap Heating on the Space Shuttle", AIAA/ASME 3rd Joint Thermophysics, Fluids, Plasma and Heat Transfer Conference, AIAA Paper 82-0840, St. Louis, MO, 1982.

${ }^{3}$ Hinderks, M., Radespiel, R., and Gülhan, A., "Simulation of Hypersonic Gap Flow with Consideration of Fluid Structure Interaction", 34th AIAA Fluid Dynamics Conference and Exhibit, AIAA Paper 2004-2238, Portland, OR, 2004.

${ }^{4}$ Hinderks, M., and Radespiel, R., "Investigation of Hypersonic Gap Flow of a Reentry Nosecap with Consideration of Fluid structure Interaction", 44th AIAA Aerospace Sciences Meeting and Exhibit, AIAA Paper 2006-0188, Reno, NV, 2006.

${ }^{5}$ Bertin, J. J., and Goodrich, W. D., "Aerodynamic Heating for Gaps in Laminar and Trasitional Boundary Layers", 18th AIAA Aerospace Sciences Meeting and Exhibit, AIAA Paper 80-0287, Pasadena, CA, 1980.

${ }^{6}$ Scott, C. D., and Maraia, R. J., "Gap Heating with Pressure Gradients", 14th AIAA Thermophysics Conference, AIAA Paper 79-1043, Orlando, FL, 1979.

${ }^{7}$ Smith, D. M., Petley, D. N., Edwards, C. L. W., and Patten, A. B., "An Investigation of Gap Heating Due to Stepped Tiles in Zero Pressure Gradient Regions of the Shuttle Orbiter Thermal Protection System", 21st AIAA Aerospace Sciences Meeting and Exhibit, AIAA Paper 83-0120, Reno, NV, 1983.

${ }^{8}$ Charbonnier, J., and Boerrigter, H., "Contribution to the Study of Gap Induced Boundary Layer Transition in Hypersonic Flow", AIAA/DGLR 5th International Aerospace Planes and Hypersonics Technologies Conference, AIAA Paper 93-5111, Munich, Germany, 1993.

${ }^{9}$ Dunavant, J. C., and Throchmorton, D. A., "Aerodynamic Heat Transfer to RSI Tile Surfaces and Gap Intersections", Journal of Spacecraft and Rockets, Vol. 11, No. 6, 1974, pp. 437-440.

${ }^{10}$ Higdon, J. J. L., "Stokes Flow in Arbitrary Two-Dimensional Domains: Shear Flow over Ridges and Cavities", Journal of Fluid Mechanics, Vol. 159, 1985, pp. 195-226.

${ }^{11}$ Traineau, J. C., Thivet, F., Gulhan, A., Cosson, E., Smith, A., and Marraffa, L., "Synthesis of the Gap Heating Analysis of the Hyflex Flight", In Proceedings of the Fifth European Symposium on Aerothermodynamics for Space Vehicles, November 8-11, 2005.

${ }^{12}$ Paolicchi, L. T. L. C., and Santos, W. F. N., "Direct Simulation Calculations of Rarefied Hypersonic Gap Flow", 3rd Southern Conference on Computational Modeling, Rio Grande, RS, Brazil, 2009.

${ }^{13}$ Bird, G. A., Molecular Gas Dynamics and the Direct Simulation of Gas Flows, Oxford University Press, Oxford, England, UK, 1994.

${ }^{14}$ Bird, G. A., "Monte Carlo Simulation in an Engineering Context," in Progress in Astronautics and Aeronautics: Rarefied gas Dynamics, edited by Sam S. Fisher, Vol. 74, part I, AIAA New York, 1981, pp. 239-255.

${ }^{15}$ Bird, G. A., "Perception of Numerical Method in Rarefied Gasdynamics," in Rarefied gas Dynamics: Theoretical and Computational Techniques, edited by E. P. Muntz, and D. P. Weaver and D. H. Capbell, Vol. 118, Progress in Astronautics and Aeronautics, AIAA, New York, 1989, pp. 374-395.

${ }^{16}$ Borgnakke, C. and Larsen, P. S., "Statistical Collision Model for Monte Carlo Simulation of Polyatomic Gas Mixture," Journal of computational Physics, Vol. 18, No. 4, 1975, pp. 405-420.

${ }^{17}$ Boyd, I. D., "Analysis of Rotational Nonequilibrium in Standing Shock Waves of Nitrogen," AIAA Journal, Vol. 28, No. 11, 1998, pp. 1997-1999.

${ }^{18}$ Bird, G. A. "A Comparison of Collision Energy-based and Temperature-based Procedures in DSMC," 26th International Symposium on Rarefied Gas Dynamics, edited by T. Abe, American Institute of Physics, 2009, pp. 245-250.

${ }^{19}$ Alexander, F. J., Garcia, A. L., and, Alder, B. J., "Cell Size Dependence of Transport Coefficient in Stochastic Particle Algorithms," Physics of Fluids, Vol. 10, No. 6, 1998, pp. 1540-1542.

${ }^{20}$ Alexander, F. J., Garcia, A. L., and, Alder, B. J., "Erratum: Cell Size Dependence of Transport Coefficient is Stochastic Particle Algorithms," Physics of Fluids, Vol. 12, No. 3, 2000, pp. 731-731.

${ }^{21}$ Garcia, A. L., and, Wagner, W., "Time Step Truncation Error in Direct Simulation Monte Carlo", Physics of Fluids, Vol. 12, No. 10, 2000, pp. 2621-2633.

${ }^{22}$ Hadjiconstantinou, N. G., "Analysis of Discretization in the Direct Simulation Monte Carlo", Physics of Fluids, Vol. 12, No. 10, 2000, pp. 2634-2638.

${ }^{23}$ Paolicchi, L. T. L. C., "Computational Analysis of Gap Effects on the Surface of Reentry Space Vehicles", MS Thesis, National Institute for Space Research (INPE), Brazil, 2010.

${ }^{24}$ Everhart, J. L., Alter, S. J., Merski, N. R., Wood, W. A., and Prabhu, R. K., "Pressure Gradient Effects on Hypersonic Cavity Flow Heating", 44th AIAA Aerospace Sciences Meeting and Exhibit, AIAA Paper 2006-0185, Reno, NV, 2006. 